\title{
Q-HeteLearn: A Progressive Learning approach for Classifying Meta-Paths in Heterogeneous Information Networks
}

\author{
Sadhana Kodali ${ }^{1}$, Madhavi Dabbiru ${ }^{2}$, B Thirumala Rao ${ }^{3}$ \\ ${ }^{1}$ PhD Scholar, Department of Computer Science and Engineering, Koneru Lakshmaiah Education \\ Foundation, Vaddeswaram, AP, India. \\ ${ }^{2}$ Professor, Department of Computer Science Engineering, Dr.L.Bullayya College of Engineering for \\ Women, Visakhapatnam, Andhra Pradesh, India. \\ 3. Professor, Department of Computer Science and Engineering, Koneru Lakshmaiah Education Foundation, \\ Vaddeswaram, AP, India.
}

\begin{abstract}
Reinforcement learning is a machine learning paradigm which has a number of applications in gaming, stock prediction, robot navigation etc. The reinforcement learning can be applied to complex real-world tasks which have adjustable problem spaces. In this paper a novel approach called Q-HeteLearn a Progressive Learning method is introduced to classify the objects by traversing the meta-paths in the Heterogeneous Information Networks. The proposed approach showed best results when compared to a traditional learning strategy called the Q-Learning and also the comparative study showed a better result with Deep Q-Learning. The concept of Q-HeteLearn which is a Progressive Learning technique is introduced to improve the swift traversal of the objects in the meta-paths and to classify them.
\end{abstract}

Keywords: Reinforcement learning, , Q-Learning, Heterogeneous Information Networks.

\section{INTRODUCTION}

The objects in the real world are heterogeneous and often associated by a semi-structured semantic relationship which we call as heterogeneous information network [1] examples include biological networks, bibliographic networks ,social networks ,highway networks[2]etc. By observing these semantically connected links between objects, we can get consequential meta-paths[3]that help us take significant decisions. Traversing these gigantic networks $[4,5]$ and learning the relationships between them using traditional approaches is very time taking and involves a lot of effort. Breaking down these gigantic interconnected objects to semi-structured information networks can be possible by classifying the interconnecting objects which are responsible to form the meta-path is the main aim of this paper. The authors implemented the QHeteLearn which is a progressive learning approach that has faster computation performance when compared with one of the traditional reinforcement learning algorithm called Q-Learning. The proposed approach is also compared with Deep Q-Learning. This paper is organized as follows: In section 2 the literature survey is provided, section 3 the proposed methodology is

discussed, section 4 gives the experimentation results and concluded in section 5 .

\section{LITERATURE SURVEY}

The Q-Learning [6]algorithm is one of the reinforcement learning technique and a model free learning strategy. QLearning is a policy based approach which tells an agent the next action to be taken at the corresponding circumstances. Q-Learning has many applications one of which includes the addressing of autonomous mobile robot navigation problem[7],in which the authors projected an improved Q-Learning algorithm for the navigation of a robot using a non-repeated state-action trajectory. The agents learned from reinforcement which has absolute value. The co-operative Q-Learning exhibited better performance than traditional Q-leaning in the context of a segmented world. In the traditional Q-Learning approach the Qvalues are infinitely updated for few steps. The condition to terminate is to check the difference in the Qvalues obtained from the successive iterations. The drawback of this approach is the time complexity. The other case of Q-Learning is named as extended Q-Learning [8], the best action state is stored. The limitation of storing the best action state may not permit another updated state.In [9] the authors used an approach to address the reinforcement learning for continuous actions. The authors in [10] used a disease prediction attack for asthma using QLearning. But the model constructed in the above technique made use of historical. In [11] the authors used the Q-Learning approach to model the energy consumption by integrating Q-Learning with radio networks which showed an improved levels of energy consumption. QLearning is also applied for home energy management using fuzzy reasoning in [12]. The Q-HeteLearn Progressive Learning approach is experimented on an image dataset to classify the images. 


\section{METHODOLOGY}

The proposed approach is appropriate for stochastic transition problems like growth of bacteria, fluctuating electric current and so on. On the other hand, in a practical application the function approximation may vary and another overhead exists with the speed of learning. In the traditional Q-Learning algorithm, The major task is to accomplish the goal state where in for every action(change from current state to new state)there is a reward and it helps in attainment of the goal state. At this point if the system is capable to progress in the accurate direction (closer to the goal state), then a certain weight is to be associated along with the feedback component. If the QSystem is unsuccessful to arrive at the goal state then a negative signal should be communicated so as to make the Q-System change its progression path. The weight can be a probability component $d$ may vary with the closeness to the goal state. Thus, the progression factor helps in accelerating the Q-algorithm in reaching its goal state swiftly. In the Q-HeteLearn approach a progression factor is defined by the symbol $F$.

$\mathrm{F}=\operatorname{diff}(\mathrm{Qgoal}, \mathrm{Q}) * \mathrm{P}(\mathrm{A}, \mathrm{W})(1)$

In the equation (1) Qgoal is the expected goal state and Q is the current state. The progression factor $F$ gives $\delta$ difference. $\mathrm{P}(\mathrm{A}, \mathrm{W})$ is the probability of our taking action $\mathrm{a}_{\mathrm{i}} \mathrm{in}$ state $\mathrm{s}_{\text {cur }}$ to choose another near state $\mathrm{s}_{\text {next }}$

$$
P(A i, w)=\frac{\exp (w i)}{\sum_{j} \exp (w j)}(2)
$$

Where exp is the expected weightage to the change of state from $s_{\text {cur }}$ to $s_{\text {next }}$. The equation in (2) indicates weighted probability $P\left(\mathrm{~A}_{\mathrm{i}}, \mathrm{w}\right)$ is the number of ways to achieve the desired outcome divided by the number of total possible outcomes. The performance of the algorithm is enhanced if this $\delta$ meets the threshold range $\mp$. When the $\operatorname{diff}($ Qgoal,Q) is calculated if it doesn't fall in the threshold range $\mathrm{F}$, which indicates that the current state $\mathrm{Q}$ is isolated from the goal state and it can be bypassed as there is nothing much to be trained from this state. According to the QHeteLearn Progressive Learning approach the threshold value is computed to reach the goal state. Traversing all the states may not accomplish to reach the goal state. To drop off the time complexity the progression factor is computed at every state and checked with the threshold. If the value is in the range of the threshold value, the state can be considered to be closer to the goal state. The PTable for the target state is set initially taking a query image and converting this query image to an array. The Pgoal state for this image is computed. The images in the folder are compared with this goal state and the similar images are identified.
Q-HeteLearn :Progressive Learning approach

Step:1Initialization:

I=Read the target image;

Convert Ito an array.

Pgoal initialised to No of zeros equal to the shape of the target image.

diff initialised to No of zeros equal to the shape of the target image.

Step 2:

Repeat for every image \{

Repeat for I.shape()

\{

Pgoal[i][j] $=\mathrm{I}[\mathrm{i}][\mathrm{j}]+\alpha^{*}\left(\mathrm{rw}+\gamma^{*}(\mathrm{I} 2[\mathrm{i}][\mathrm{j}])-\mathrm{I}[\mathrm{i}][\mathrm{j}]\right)$

Compute the difference for the target image and every image in the folder cv2.subtract(I,Pgoal)

Estimate the progression factor:

$$
\mathrm{F}=\operatorname{diff}(\mathrm{Pgoal}, \mathrm{I}) * \mathrm{P}\left(\mathrm{A}_{\mathrm{i}}, \mathrm{w}\right)
$$

If $\mathrm{F}<=\mathrm{F}$

Update Ptable

continue

else

skip state and goto next;

\}

\}

Figure 1: Q-HeteLearn approach.

The Figure 1 represents an algorithm which explains the proposed methodology.

\section{EXPERIMENTATION}

A stochastic process changes over time and so is the plant growth and the size, colour of the leaf changes with time, climate and various other conditions. If the leaf is observed as a set of numbers represented in a two-dimensional array, then the array of a healthy leaf indicates different set of points while the diseased leaf indicates a different number in the given array. When we observe a spotted leaf and compare it with the goal state, where the goal state is that of a healthy leaf, the action taken should be different recommendations of pest control to reduce the plant disease. The dataset to do the experimentation is obtained from [13]. This is an image repository of different plants and their corresponding diseases. The plant village image dataset contains 20639 images. The foremost step to this approach is the data pre-processing step. Pre-process the images by comparing them using cv2.subtract () method then set a threshold value $\mathrm{F}$, If the threshold value constraint is not set then it is a completely different image. The experimentation is carried on an Intel core 2 duo processor with 4GB Memory. The code for implementing the Progressive Learning algorithm is written using python. 
The Table 1 and graph in Figure 2 give explanation about the time taken for execution of the number of images corresponding to Q-Learning and Q-HeteLearn Progressive Learning approach. From the graph it can be observed that the time taken to execute in the Q-HeteLearn approach is comparatively less. The Q-Learning approach takes more time as the number of images increases considerably. From the 997 images 396 images are used for test and residual 601 images are considered for training.

Table 1: Runtimes of Q-Learning and Q-HeteLearn Progressive Learning.

\begin{tabular}{|l|l|l|}
\hline $\begin{array}{l}\text { No of } \\
\text { Images } \\
\text { in the } \\
\text { folder }\end{array}$ & $\begin{array}{l}\text { Q- } \\
\text { Learning } \\
\text { Execution } \\
\text { time }\end{array}$ & $\begin{array}{l}\text { Q- } \\
\text { HeteLearn } \\
\text { Execution } \\
\text { time }\end{array}$ \\
\hline 16 & $2: 75 \operatorname{secs}$ & $2: 56 \operatorname{secs}$ \\
\hline 35 & $4: 32 \mathrm{secs}$ & $2: 75 \mathrm{secs}$ \\
\hline 45 & $5: 46 \mathrm{secs}$ & $2: 97 \mathrm{secs}$ \\
\hline 59 & $7: 26 \mathrm{secs}$ & $3.46 \mathrm{secs}$ \\
\hline
\end{tabular}

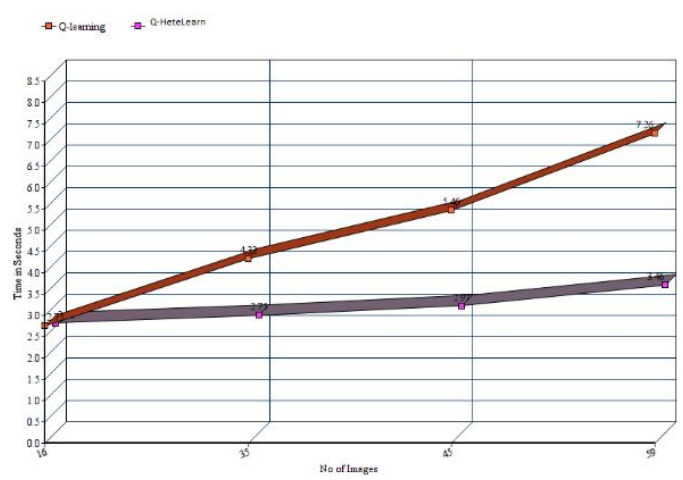

Figure 2: Graph used to represent the runtime of QLearning and Q-HeteLearn.

Table 2: Runtime results

\begin{tabular}{|l|l|l|}
\hline $\begin{array}{l}\text { Number } \\
\text { of Images }\end{array}$ & Q-Learning & Q-HeteLearn \\
\hline 997 & $5.46 \mathrm{mins}$ & $28 \mathrm{Sec}$ \\
\hline
\end{tabular}

From Table 2 we can examine that the time to execute is less than a minute in case of 997 images which contain different classes. The target image matches only with a few images and this algorithm quickly prunes the images that do not match the threshold value.

\subsection{Comparison with Deep Q-Learning:}

Q-Learning has certain limitations that it works better for smaller environments with discrete, finite state and action spaces. But a meta-path expands as new objects add to it and new relationships exist. Therefore, the Deep QLearning strategy is also used to compare Q-HeteLearn. The Deep Q-Learning makes use of the neural network which approximates the values which are unstable and diverging. Adjusting the weights in the neural networks include back propagation.In Deep Q-Learning a small update to $\mathrm{Q}$ has significant change in the policy. These variable changes are updated and managed by experience replay and iterative updates which increases the time complexity when compared to Q-HeteLearn which took minimal time to learn about the meta-paths. We compare Deep Q-Learning with Q-HeteLearn by already available results in [14] the input of Deep Q-Learning is the pixels and game scores. The input of Q-HeteLearn is an image which is converted to an array of pixels. The state and actions are obtained by playing a game 30 times for 5 minutes with an $\square=0.05$. The value of $\gamma=0.99$ is set for Deep Q-Learning and $\gamma=0.9$ for Q-HeteLearn. The Deep QLearning uses the deep neural networks to categorize the objects from raw sensory data whereas the Q-HeteLearn traverses the meta-paths and applies a progression factor to categorize objects.

\section{CONCLUSION}

In this paper a Progressive reinforcement learning approach called the Q-HeteLearn is devised for classification of meta-paths in heterogeneous information networks .This approach uses a progression factor for QLearning for improving the execution time. The QHeteLearn shows a better performance with regard to runtime when compared with the traditional Q-Learning as well as the latest algorithm deep Q-Learning.As a part of the future work, the deep learning techniques will be used to propose recommendations in heterogeneous information networks without clipping the rewards and by optimizing the P table.

\section{REFERENCES}

1. Chuan Shi,Yitong Li,Jiawei Zhang,Yizhou Sun, PhilipSYu,A survey of heterogeneous information network analysis IEEE transactions on Knowledge and Data Engineering", Volume 29,Issue 1,Jan 12017. https://doi.org/10.1109/TKDE.2016.2598561

2. Srivastava, Rupesh \& Greff, Klaus \& Schmidhuber, Networks.

3. Yizhou Sun,Jiawei Han,Xifeng Yan,Philip S. Yu ,Tianyi Wu, PathSim: Meta Path-Based Top-K Similarity Search in Heterogeneous Information Networks Proceedings of the VLDB endowmwent, Vol 4 , No 11,September 2011.

4. B.Tirapathi Reddy, M.V.P Chandra Sekhara Rao Filter Based Data Deduplication in Cloud Storage using Dynamic Perfect Hash Functions International Journal of Simulation Systems, Science \&amp; Technology, and ISSN: 1473804x online, 1473-8031print. DOI 10.5013/IJSSST.a.19.04.08. 2018 
5. B.Tirapathi Reddy, Dr.M.V.P. Chandra Sekhara Rao, Performance evaluation of various data deduplication schemes in cloud storage, IJPAM,Sep-2016,Vol. 116, Issue No.5,175-180.

6. Christopher J.C.H.Watkins Technical Note QLearning Machine Learning, 8,279-292(1992). https://doi.org/10.1023/A:1022676722315

7. Jawad Muhammad, Ihsan ÖmürBucak An improved Q-Learning algorithm for an autonomous mobile robot navigation problem International Conference on Technological Advances in Electrical, Electronics and Computer Engineering, May 2013.

8. V.Borkar Q-Learning for Risk-Sensitive control Mathematics of Operations Research27(2),294311,2002 . https://doi.org/10.1287/moor.27.2.294.324

9. Jose del R Millan,Daniele posenato,Eric Dedieu Continuous-Action Q-Learning, Machine Learning Volume 49 Issue pp-247-265, November 2002.

10. Q. Do, S. Tran and A. Doig, Reinforcement Learning Framework to Identify Cause of Diseases - Predicting Asthma Attack Case 2019 IEEE International Conference on Big Data (Big Data), Los Angeles, CA, USA, 2019, pp. 4829-4838

11. Mohammed saleh, Bendella, Badr Benmammr, Francine Krief Using cognitive radio to deliver Green Communication International Journal of Knowledge Engineering and Data Mining, Vol 6,Issue 4. February 2020.

12. Fayiz alfaver, M Danal,Y Sun Demand Response Strategy Based on Reinforcement Learning and Fuzzy Reasoning for Home Energy Management IEEE Access February 2020. https://doi.org/10.1109/ACCESS.2020.2974286

13. https://github.com/spMohanty/PlantVillageDataset

14. Volodymyr Mnih, Koray Kavukcuoglu , David Silver et.al Human-level control through deep reinforcement learning NATURE, VOL 518,26 February 2015.

https://doi.org/10.1038/nature14236 